Bull. Austral. Math. Soc.

$44 \mathrm{~A} 10,44 \mathrm{~A} 40,46 \mathrm{~F} 99$

VoL. 46 (1992) [347-352]

\title{
THE LAPLACE TRANSFORM ON A CLASS OF BOEHMIANS
}

\section{DENNIS NEMzER}

The one-sided Laplace transform is defined on a space of generalised functions called transformable Boehmians. The space of one-sided Laplace transformable distributions is shown to be a proper subspace of transformable Boehmians. Some basic properties of the Laplace transform are investigated. An inversion formula and an Abelian theorem of the final type are obtained.

\section{INTRODUCTION AND PRELIMINARIES}

A relatively new class of generalised functions, called Boehmians, has opened the door to new areas of research (see $[4,5,6,7,8,9])$. The class of all Boehmians is quite general. Indeed, the space of all Schwartz distributions on the real line (circle) can be identified with a proper subspace of Boehmians $[4,8]$. There are Boehmians which are not functions that satisfy Laplace's equation $u_{x x}+u_{y y}=0$ [6]. Also, there are periodic Boehmians which are not periodic hyperfunctions [9].

In this note, we define the one-sided Laplace transform on a subclass of Boehmians (which is also a subclass of regular Mikusinski operators [2]) and investigate some of its properties.

Let $\Omega$ be a subset of the real line $R$. The space of all continuous complex-valued functions on $\Omega$ will be denoted by $C(\Omega)$. The space of all functions $f \in C(R)$ such that $f(t)=0$ for $t<0$ will be denoted by $C_{+}(R)$. The support of a continuous function $f$, denoted by supp $f$, is the complement of the largest open set on which $f$ is zero. The convolution product of two functions $f, g \in C_{+}(R)$ is given by $(f * g)(t)=$ $\int_{0}^{t} f(t-u) g(u) d u$.

A sequence of continuous nonnegative functions $\left\{\varphi_{n}\right\}$ will be called a delta sequence if

(i) $\int_{-\infty}^{\infty} \varphi_{n}(t)=1$ for $n=1,2, \ldots$; and

(ii) $\operatorname{supp} \varphi_{n} \subseteq\left[0, \varepsilon_{n}\right], \varepsilon_{n} \rightarrow 0$ as $n \rightarrow \infty\left(\varepsilon_{n}>0\right)$.

The following easily proved result will be needed. If $f$ is a continuous function and $\left\{\varphi_{n}\right\}$ is a delta sequence, then $f * \varphi_{n} \rightarrow f$ uniformly on compact sets as $n \rightarrow \infty$.

A pair of sequences $\left(f_{n}, \varphi_{n}\right)$ is called a quotient of sequences, if $f_{n} \in C_{+}(R)$ for $n=1,2, \ldots,\left\{\varphi_{n}\right\}$ is a delta sequence, and $f_{k} * \varphi_{m}=f_{m} * \varphi_{k}$ for all $k$ and

Received 11 October 1991

Copyright Clearance Centre, Inc. Serial-fee code: 0004-9729/92 \$A2.00+0.00. 
$m$. Two quotients of sequences $\left(f_{n}, \delta_{n}\right)$ and $\left(g_{n}, \sigma_{n}\right)$ are said to be equivalent if $f_{k} * \sigma_{m}=g_{m} * \delta_{k}$ for all $k$ and $m$. A straightforward calculation shows that this is an equivalence relation. The equivalence classes are called Boehmians. The space of all Boehmians will be denoted by $\beta$ and a typical element of $\beta$ will be written as $x=f_{n} / \delta_{n}$. For a more general construction of Boehmians see [4].

By defining a natural addition, multiplication and scalar multiplication on $\beta$, that is $f_{n} / \delta_{n}+g_{n} / \sigma_{n}=\left(f_{n} * \sigma_{n}+g_{n} * \delta_{n}\right) / \delta_{n} * \sigma_{n}, f_{n} / \delta_{n} * g_{n} / \sigma_{n}=f_{n} * g_{n} / \delta_{n} * \sigma_{n}$, and $\alpha f_{n} / \delta_{n}=\alpha f_{n} / \delta_{n}$ where $\alpha$ is a complex number, $\beta$ becomes a convolution algebra.

Since the Boehmian $\delta_{n} / \delta_{n}$ corresponds to the Dirac delta distribution, we denote it by $\delta$. Moreover, the $n$th derivative of $\delta$ is given by the formula $D^{n} \delta=\delta^{(n)}=\delta_{k}^{(n)} / \delta_{k}$, where $\left\{\delta_{n}\right\}$ is any infinitely differentiable delta sequence. In general, the $n$th derivative of $x \in \beta$ is given by $D^{n} x=x * \delta^{(n)}$.

By the translation operator on $C_{+}(R)$, we mean the operator $\tau_{\alpha}, \alpha$ real, such that $\left(\tau_{\alpha} f\right)(t)=f(t-\alpha)$ for $-\infty<t<\infty$. The translation operator can be extended to an element $x=f_{n} / \delta_{n} \in \beta$ by $\tau_{\alpha} x=\tau_{\alpha} f_{n} / \delta_{n}$.

A sequence $\left\{x_{n}\right\}$ of Boehmians is said to converge to the Boehmian $x$, denoted by $\delta-\lim x_{n}=x$, if there exists a delta sequence $\left\{\delta_{n}\right\}$ such that for each $n$ and $k$, $x_{n} * \delta_{k}, x * \delta_{k} \in C_{+}(R)$ and for all $k,\left(x_{n}-x\right) * \delta_{k} \rightarrow 0$ uniformly on compact sets as $n \rightarrow \infty$.

Since convolution is a continuous operation on $\beta$, if $\delta-\lim x_{n}=x$ then $\delta-\lim D^{m} x_{n}=D^{m} x$ for $m=0,1,2, \ldots$

\section{The Laplace transform}

A Boehmian $x$ is said to be transformable if there exists a delta sequence $\left\{\delta_{n}\right\}$ and a nonnegative number $\alpha$ such that $x * \delta_{n} \in C_{+}(R)$ for all $n$ and $x * \delta_{n}=O(\exp (\alpha t))$ as $t \rightarrow \infty$ for all $n$. The space of all transformable Boehmians will be denoted by $\beta_{L}$.

A Laplace transformable distribution $f \in \mathcal{L}_{+}[11]$ can be identified with the Boehmian $f * \delta_{n} / \delta_{n} \in \beta_{L}$ where $\left\{\delta_{n}\right\}$ is any delta sequence. Indeed, by using the representation

$$
f=\sum_{i=0}^{k} c_{i}(d / d t)^{i}\left[\exp (\alpha t) G_{i}(t)\right]
$$

where for each $i, c_{i}$ is a complex number $G_{i} \in C[0, \infty)$ and $G_{i}=O\left(t^{m}\right)$ as $t \rightarrow \infty$ (for some $m$ ) [10], we see that $f * \delta_{n} \in C_{+}(R)$ and $f * \delta_{n}=O(\exp (\alpha t))$ as $t \rightarrow \infty$ for all $n$. Thus $\mathcal{L}_{+}$can be viewed as a subspace of $\beta_{L}$. The space $\mathcal{L}_{+}$is actually a proper subspace of $\beta_{L}$ (see Example 2.6).

If $f \in C_{+}(R)$ such that $f(t)=O(\exp (\alpha t))$ as $t \rightarrow \infty$, for some real number $\alpha$, then the Laplace transform of $f$ is given by $F(z)=\mathcal{L}[f]=\int_{0}^{\infty} \exp (-z t) f(t) d t$ for 
Re $z>\alpha$. Now, for $x \in \beta_{L}$ where $x * \delta_{n} \in C_{+}(R)$ and $x * \delta_{n}=O(\exp (\alpha t))$ as $t \rightarrow \infty$ for all $n$, the Laplace Transform $\mathcal{L}[x]$ of $x$ is defined by the equation $\mathcal{L}[x] \mathcal{L}\left[\delta_{n}\right]=\mathcal{L}\left[x * \delta_{n}\right]$ for all $n$.

By using the Convolution Theorem for Laplace transforms and the fact that if $\left\{\delta_{n}\right\}$ is a delta sequence then for every complex number $z, \mathcal{L}\left[\delta_{n}\right](z) \rightarrow 1$ as $n \rightarrow \infty$, we see that the Laplace transform for each transformable Boehmian is well-defined. Moreover, for each $x \in \beta_{\boldsymbol{L}}$ there exists a half-plane in which $\mathcal{L}[x]$ is an analytic function.

Now, if $f \in \mathcal{L}_{+}$then $f$ has a Laplace transform as a member of $\mathcal{L}_{+}$and as a member of $\beta_{L}$. By using the representation (2.1) for $f$ it follows that the two definitions for the Laplace transform agree on $\mathcal{L}_{+}$.

As the next theorem shows, many of the standard properties concerning the ordinary Laplace transform are preserved. Since the proof follows directly from the definitions and the properties of the ordinary Laplace transform, it is omitted.

THEOREM 2.1. For $x, y \in \beta_{L}$, if $\mathcal{X}(z)$ and $\mathcal{Y}(z)$ are the Laplace transforms of $x$ and $y$ respectively, then:

1. $\mathcal{L}[x+y]=\mathcal{X}(z)+\mathcal{Y}(z)$.

2. $\mathcal{L}[\alpha x]=\alpha \mathcal{X}(z), \alpha$ a complex number.

3. $\mathcal{L}\left[D^{n} x\right]=z^{n} \mathcal{X}(z)$.

4. $\mathcal{L}[x * y]=\mathcal{X}(z) \mathcal{Y}(z)$.

5. $\mathcal{L}\left[\tau_{\alpha} x\right]=\exp (-\alpha z) \mathcal{X}(z), \alpha$ a real number.

6. (Uniqueness Theorem) If $\mathcal{X}(z)=0$, then $x=0$.

Theorem 2.2. (Inversion Formula) For each $x \in \beta_{L}, x=\delta-\lim 1 / 2 \pi i$ $\int_{\gamma-i \infty}^{\gamma+i \infty} \exp (z t) \mathcal{L}\left[x * \delta_{n}\right](z) d z$, where $\left\{\delta_{n}\right\}$ is any delta sequence such that for each $n$, $x * \delta_{n} \in C_{+}^{\infty}(R)$ and $x * \delta_{n}=O(\exp (\alpha t))$ as $t \rightarrow \infty$, and $\gamma$ is any real number such that $\gamma>\alpha$.

Proof: The existence of a delta sequence such that for each $n, x * \delta_{n} \in C_{+}^{\infty}(R)$ and $x * \delta_{n}=O(\exp (\alpha t))$ as $t \rightarrow \infty$ is not as restrictive as it first appears. Indeed, suppose for each $n, x * \varphi_{n} \in C_{+}(R)$ and $x * \varphi_{n}=O(\exp (\alpha t))$ as $t \rightarrow \infty$ for some delta sequence $\left\{\varphi_{n}\right\}$. Let $\left\{\psi_{n}\right\}$ be any infinitely differentiable delta sequence (that is $\psi_{n} \in C_{+}^{\infty}(R)$ for all $n$ ). Then the delta sequence $\left\{\delta_{n}\right\}$, where $\delta_{n}=\varphi_{n} * \psi_{n}$ for all $n$, will be the desired delta sequence.

Now, since for each $n, x * \delta_{n} \in C_{+}^{\infty}(R)$ and $x * \delta_{n}=O(\exp (\alpha t))$ as $t \rightarrow \infty$, we have that for each integer $k$ and for all $n$, the function $\mathcal{L}\left[x * \delta_{n}\right](z) /(1+|z|)^{k}$ is bounded in the half-plane $\operatorname{Re} z \geqslant \gamma$, where $\gamma>\alpha$. Hence, for $n=1,2, \ldots$, $\left(x * \delta_{n}\right)(t)=1 /(2 \pi i) \int_{\gamma-i \infty}^{\gamma+i \infty} \exp (z t) \mathcal{L}\left[x * \delta_{n}\right](z) d z$, for any $\gamma>\alpha$ (see [3]). Now, for $k=1,2, \ldots,\left(x * \delta_{n}\right) * \delta_{k}=\left(x * \delta_{k}\right) * \delta_{n} \rightarrow x * \delta_{k}$ uniformly on compact sets as $n \rightarrow \infty$. 
That is, $\delta-\lim \left(x * \delta_{n}\right)=x$ and the result is established.

Definition 2.3: Let $\Omega$ be an open subset of $R$. A Boehmian $x$ is said to be equal to a continuous function $f$ on $\Omega$, denoted by $x=f$ on $\Omega$, if there exists a delta sequence $\left\{\delta_{n}\right\}$ such that $x * \delta_{n} \in C(R)$ for all $n$ and $x * \delta_{n} \rightarrow f$ uniformly on compact subsets of $\Omega$ as $n \rightarrow \infty$.

The support of $x \in \beta$, written supp $x$, is the complement of the largest open set on which $x$ is zero. For example, given any delta sequence $\left\{\delta_{n}\right\}$ and $\varepsilon>0, \delta_{n}(t) \rightarrow 0$ uniformly for $|t|>\varepsilon$ as $n \rightarrow \infty$. Thus, $\operatorname{supp} D^{n} \delta=\{0\}$ for $n=0,1,2, \ldots$

Suppose that $x=f_{n} / \delta_{n} \in \beta$ such that $x$ has bounded support. It is not difficult to show that

$$
\operatorname{supp} f_{n} \subseteq \operatorname{supp} x+\operatorname{supp} \delta_{n} \text { for all } n
$$

and hence, for each $n$, the function $x * \delta_{n}$ is bounded. Thus we obtain the following theorem.

THEOREM 2.4. If $x \in \beta$ has bounded support, then $x \in \beta_{L}$.

THEOREM 2.5. Suppose that $\left\{x_{n}\right\}$ is a sequence of Boehmians such that $\delta-\lim x_{n}=x$ and $\operatorname{supp} x_{n} \subseteq[0, T]$ for all $n$ (for some $T \geqslant 0$ ). Then $\mathcal{X}_{n} \rightarrow \mathcal{X}$ uniformly on compact sets as $n \rightarrow \infty$.

Proof: Suppose that for each $n, \operatorname{supp} x_{n} \subseteq[0, T]$ and $\delta-\lim x_{n}=x$. Then supp $x \subseteq[0, T]$ and there exists a delta sequence $\left\{\delta_{n}\right\}$ such that for each $n$ and $k, x_{n} * \delta_{k}, x * \delta_{k} \in C_{+}(R)$ and for each $k,\left(x_{n}-x\right) * \delta_{k} \rightarrow 0$ uniformly on compact sets as $n \rightarrow \infty$. By (2.2) we may assume that for each $n$ and $k$, $\operatorname{supp}\left(x_{n} * \delta_{k}\right), \operatorname{supp}\left(x * \delta_{k}\right) \subseteq[0, T+1]$.

To complete the proof it suffices to show that for each complex number $z_{0} \in C$ there exists a neighbourhood $S(\varepsilon)(\varepsilon>0)$ for $z_{0}$ such that $\mathcal{X}_{n} \rightarrow \mathcal{X}$ uniformly on $S(\varepsilon)$ as $n \rightarrow \infty$. Let $z_{0} \in C$. Since for each $k, \mathcal{L}\left[\delta_{k}\right]$ is continuous and $\mathcal{L}\left[\delta_{k}\right]\left(z_{0}\right) \rightarrow 1$ as $k \rightarrow \infty$, there exist an integer $k_{0}$ and an $\varepsilon>0$ such that $\left|\mathcal{L}\left[\delta_{k_{0}}\right](z)\right| \geqslant 1 / 2$ for all $z \in S(\varepsilon)$. So, for each $z \in S(\varepsilon)$,

$$
\begin{aligned}
\left|\mathcal{X}_{n}(z)-\mathcal{X}(z)\right| & =\left|\mathcal{L}\left[x_{n} * \delta_{k_{0}}\right](z) / \mathcal{L}\left[\delta_{k_{0}}\right](z)-\mathcal{L}\left[x * \delta_{k_{0}}\right](z) / \mathcal{L}\left[\delta_{k_{0}}\right](z)\right| \\
& \leqslant 2\left|\mathcal{L}\left[x_{n} * \delta_{k_{0}}\right](z)-\mathcal{L}\left[x * \delta_{k_{0}}\right](z)\right|
\end{aligned}
$$

Now, since for each $n, \operatorname{supp}\left(x_{n} * \delta_{k_{o}}\right), \operatorname{supp}\left(x * \delta_{k_{0}}\right) \subseteq[0, T+1]$ and $\left(x_{n}-x\right) * \delta_{k_{0}} \rightarrow 0$ uniformly on compact sets as $n \rightarrow \infty, \mathcal{L}\left[x_{n} * \delta_{k_{0}}\right] \rightarrow \mathcal{L}\left[x * \delta_{k_{0}}\right]$ uniformly on compact sets as $n \rightarrow \infty$. The proof is completed by applying (2.3) to the above. 
It is well-known that if $f \in \mathcal{L}_{+}$, then there exist a real number $\alpha$ and a polynomial $P$ such that $F(z)$ is analytic in the half-plane $\operatorname{Re} \geqslant \alpha$ and $|F(z)| \leqslant P(|z|)$ for $\operatorname{Re} z \geqslant \alpha$ [11]. Thus, the following example demonstrates that $\mathcal{L}_{+}$is a proper subspace of $\beta_{L}$.

EXAMPLE 2.6: Since for each $\alpha>1$ the Denjoy-Carleman class $C_{I}\{1 / \Gamma(1+\alpha n)\}$ $\left(\Gamma(t)=\int_{0}^{\infty} \exp (-u) u^{t-1} d u\right)$ is not quasi-analytic, $x_{\alpha}=\sum_{n=0}^{\infty} D^{n} \delta / \Gamma(1+\alpha n) \in \beta$ (see [1]). Now, for $\alpha>1, \operatorname{supp} x_{\alpha}=\{0\}$ (see [1]), thus $x_{\alpha} \in \beta_{L}$. Moreover, by applying Theorems 2.1 and 2.5, for each $\alpha>1, \mathcal{L}\left[x_{\alpha}\right]=\sum_{n=0}^{\infty} z^{n} / \Gamma(1+\alpha n)$. Furthermore, $\mathcal{L}\left[x_{\alpha}\right]$ is an entire function of order $1 / \alpha$ (that is, for every $\varepsilon>0$, $\mathcal{L}\left[x_{\alpha}\right](z)=O\left(\exp \left(|z|^{1 / \alpha+\varepsilon}\right)\right)$ as $|z| \rightarrow \infty$, but this relation does not hold for any $\varepsilon<0)$.

Since a locally integrable function $f$ can be identified with the Boehmian $f * \delta_{n} / \delta_{n}$ (where $\left\{\delta_{n}\right\}$ is any delta sequence), we say that a Boehmian $x$ is equal to $f$ on an open set $\Omega$ if $x-f=0$ on $\Omega$.

For a transformable Boehmian $x$ that is equal to a well-behaved function on $(T, \infty)$ for some $T>0$, we prove an Abelian theorem of the final type. That is, we relate the asymptotic behaviour of $x$ at infinity to the asymptotic behaviour of $\mathcal{X}(z)$ at a singularity.

It is well-known [3] that if $h$ is a locally integrable function such that $\lim _{t \rightarrow \infty} h(t) \exp \left(-z_{0} t\right) / t^{\lambda}=A\left(A\right.$ and $z_{0}$ complex, $\left.\operatorname{Re} \lambda>-1\right)$, then $\mathcal{L}[h]=H(z)$ exists for $\operatorname{Re} z>\operatorname{Re} z_{0}$ and

$$
\lim _{z \rightarrow z_{0}}\left(z-z_{0}\right)^{\lambda+1} H(z) / \Gamma(\lambda+1)=A \text { for }\left|\arg \left(z-z_{0}\right)\right| \leqslant \psi<\pi / 2 .
$$

Suppose that $x=f_{n} / \delta_{n}$ is a transformable Boehmian having bounded support. By (2.2) we see that for each $n, f_{n}$ also has bounded support, and hence $F_{n}(z)$ is an entire function for all $n$. Moreover, for each $z \in C \mathcal{L}\left[\delta_{n}\right](z) \rightarrow 1$ as $n \rightarrow \infty$ and $\mathcal{L}\left[\delta_{n}\right]$ is also an entire function for all $n$. Thus, $\mathcal{X}(z)$ is an entire function and hence

$$
\lim _{z \rightarrow z_{0}}\left(z-z_{0}\right)^{\lambda+1} \mathcal{X}(z) / \Gamma(\lambda+1)=0 \quad \text { (where } \operatorname{Re} \lambda>-1, z_{0} \text { complex). }
$$

Now, suppose that $x \in \beta_{L}$ such that over some interval $T<t<\infty, x$ is equal to a locally integrable function $h$ and $\lim _{t \rightarrow \infty} h(t) \exp \left(-z_{0} t\right) / t^{\lambda}=A$ ( $A$ and $z_{0}$ complex). Then, $x=y+h$, where $y$ is a transformable Boehmian having bounded support. Applying (2.4) and (2.5) to $x=y+h$ we obtain the next theorem.

Theorem 2.7. Let $x \in \beta_{L}$ such that over some semi-infinite interval $T<$ $t<\infty, x$ is equal to a locally integrable function $h$ and $\lim _{t \rightarrow \infty} h(t) \exp \left(-z_{0} t\right) / t^{\lambda}=$ $A\left(A\right.$ and $z_{0}$ complex, $\left.\operatorname{Re} \lambda>-1\right)$. Then $\mathcal{X}(z)$ exists for $\operatorname{Re} z>\operatorname{Re} z_{0}$ and $\lim _{z \rightarrow z_{0}}\left(z-z_{0}\right)^{\lambda+1} \mathcal{X}(z) / \Gamma(\lambda+1)=A$ in $\left|\arg \left(z-z_{0}\right)\right| \leqslant \psi<\pi / 2$. 


\section{ReFeRENCES}

[1] T.K. Boehme, 'On power series in the differentiation operator', Studia Math. (1973), 309-317.

[2] T.K. Boehme, 'The support of Mikusinski operators', Trans. Amer. Math. Soc. 176 (1973), 319-334.

[3] G. Doetsch, Theorie der Laplace-transformation Band 1 (Verlag Birkhauser, Basel, 1950).

[4] P. Mikusinski, 'Convergence of Boehmians', Japan. J. Math. 9 (1983), 159-179.

[5] P. Mikusinski, 'Boehmians as generalized functions', Acta Math. Hungar. 51 (1988), 271-281.

[6] P. Mikusinski, 'On harmonic Boehmians', Proc. Amer. Math. Soc. 106 (1989), 447-449.

[7] P. Mikusinski, 'Boehmians on open sets', Acta Math. Hungar. 55 (1990), 63-73.

[8] D. Nemzer, 'Periodic Boehmians', Internat. J. Math. Math. Sci. 12 (1989), 685-692.

[9] D. Nemzer, 'Periodic Boehmians II', Bull. Austral. Math. Soc. 44 (1991), 271-278.

[10] K.R. Sonavane, 'A representation of one-sided Laplace transformable generalized functions', Indian J. Pure Appl. Math. 7 (1976), 477-481.

[11] A.H. Zemanian, Distribution theory and transform analysis (Dover Publications, New York, 1987).

Department of Mathematics

California State University, Stanislaus

Turlock California $\mathbf{9 5 3 8 0}$

United States of America 